\title{
Magnitude e métodos de perda rápida de peso em judocas de elite
}

\author{
Magnitude and methods of rapid weight loss \\ in elite judo athletes
}

Guilherme Giannini ARTIOLI ${ }^{1}$ Fernanda Baeza SCAGLIUSI ${ }^{1}$ Viviane Ozores POLACOW ${ }^{1}$ Bruno GUALANO'

Antonio Herbert LANCHA JUNIOR ${ }^{1}$

R E S U M O

\section{Objetivo}

Identificar os métodos utilizados para redução rápida de peso pré-competição, bem como determinar a prevalência da utilização de tais métodos entre judocas de elite e a magnitude da redução do peso.

\section{Métodos}

Fizeram parte do estudo 28 judocas de elite do sexo masculino, da cidade de São Paulo. Os atletas tiveram aferido o seu peso corporal, e relataram em que categorias de peso competiam. Desses, 19 foram entrevistados por nutricionista, para a investigação de questões relativas ao histórico de peso e de dietas. O estudo foi conduzido entre agosto de 2002 e junho de 2004.

\section{Resultados}

Os resultados mostraram que 85,7\% da amostra estava, em média, 4,2\% (desvio-padrão=2,6) acima do limite de peso da categoria. Entre os que foram entrevistados, $89,5 \%$ relataram que, habitualmente, mantinham o peso acima do máximo da categoria, e 100\% já pesaram mais do que tal limite. De modo geral, eles pesavam $3,7 \%$ (desvio-padrão=1,8) a mais do que o peso da categoria, e chegaram a atingir $7,8 \%$ (desvio-padrão=3\%) acima do limite. Os métodos de redução rápida de peso mais utilizados foram: desidratação, diminuição da ingestão energética e diminuição da ingestão de doces e gorduras.

\section{Conclusão}

Grande parte dos judocas avaliados apresenta peso corporal acima do limite de suas categorias e o reduz significativamente antes das competições, por meio de diversos métodos, muitos dos quais são prejudiciais à saúde e ao desempenho. Medidas para desencorajar tais práticas devem ser estudadas e implementadas como forma de prevenir conseqüênias mais graves.

Termos de indexação: artes marciais; atleta; desempenho; perda de peso; peso corporal; saúde.

\footnotetext{
1 Universidade de São Paulo, Escola de Educação Física e Esporte, Laboratório de Nutrição e Metabolismo Aplicados à Atividade Motora. Av. Professor Mello Moraes, 65, Cidade Universitária, 05508-900, São Paulo, SP, Brasil. Correspondência para/Correspondence to: A.H. LANCHA JUNIOR.
} 


\section{A B S T R A C T}

\section{Objective}

The objective of the present study was to identify the methods used to rapidly lose weight before a competition and determine the prevalence of such methods among elite judo athletes and the magnitude of weight loss.

\section{Methods}

This sample consisted of 28 male elite judo athletes from the city of São Paulo. Their body weight and the weight category in which they competed were recorded. Of these, 19 were interviewed by a nutritionist to investigate weight and diet histories. The study took place between August 2002 and June 2004.

\section{Results}

The results showed that $85.7 \%$ of the sample was, on average, $4.2 \%$ (standard deviation=2.6) above the limit of their weight categories. Among those who were interviewed, $89.5 \%$ reported that they usually kept the body weight above the limit, and 100\% reported that their weight was, at least once, above this limit. Generally, they weighed 3.7\% (standard deviation=1.8) more than the weight limit of their category, sometimes as much as $7.8 \%$ (standard deviation=3\%) above the limit. The most frequently used methods to lose weight rapidly were dehydration, decrease in energy intake and decreased consumption of sweets and fats.

\section{Conclusion}

Most of the judo athletes assessed present body weight above the limit of their categories and they lose weight quickly before competitions, using many methods, some of which are detrimental to their health and performance. Measures to discourage such practices should be studied and implemented in order to prevent more severe complications.

Indexing terms: martial arts; athletic; performance; weight loss; body weight; health.

\section{N T R O D U Ç Ã O}

Alguns atletas necessitam manter a massa corporal constantemente reduzida por exigência de suas modalidades, como no caso de dançarinos, patinadores e ginastas. Outros, como atletas de luta, para competir em categorias mais leves contra adversários menores e mais fracos, reduzem seu peso corporal dias antes das competições e o recuperam subseqüentemente ${ }^{1,2}$.

Steen \& Brownell ${ }^{2}$ relataram que, entre atletas norte-americanos universitários de luta olímpica, $41 \%$ perdem de 5 a 9,1 kg em cada semana durante uma temporada. Alguns atletas relataram diminuir $22,7 \mathrm{~kg}$, enquanto outros relataram reduzir o peso cerca de 60 vezes em uma temporada. Para tanto, esses atletas utilizam uma série de procedimentos que podem tanto prejudicar o desempenho quanto a saúde ${ }^{1,3-8}$. Entre as estratégias mais utilizadas para diminuir o peso estão: restrição alimentar severa, realização de exercícios intensos, e desidratação - alcançada pela restrição da ingestão de líquidos, pelo uso de saunas, e pelo treinamento em ambientes quentes com ou sem roupas de plástico e borracha ${ }^{1,2,9,10}$. Alguns relatos apontam que até indução de vômitos e ingestão de laxativos e diuréticos são adotados, na tentativa de alcançar o peso da categoria².

Para que se tenha um bom desempenho em torneios de judô, é necessário que o atleta possua elevado nível técnico-tático, bem como força, capacidade aeróbia, flexibilidade, potência e resistência anaeróbia ${ }^{11}$. Em competições de alto nível, nas quais o desenvolvimento técnico-tático dos atletas é bastante equivalente, a importância de preparação física adequada torna-se ainda mais evidente, e pequenas alterações, em qualquer variável que influencie o desempenho, podem determinar o resultado final de uma luta ou competição. Desse modo, a prática da redução rápida de peso pode ser contraproducente para o desempenho esportivo, e atletas brasileiros podem estar competindo com certo comprometimento de seu potencial físico.

Uma vez que a maioria dos estudos sobre redução rápida de peso foi feita em lutadores 
norte-americanos de luta olímpica, são necessárias mais investigações sobre a magnitude desse problema entre judocas brasileiros, para que se tenha base científica que fundamente condutas adequadas à correção de tais práticas.

Nesse sentido, este estudo teve como objetivos analisar quantitativamente a inadequação do peso corporal em relação ao peso das categorias, em atletas de judô; identificar os métodos de perda de peso pré-competição adotados por esses atletas; e analisar qualitativamente esses métodos, apontando soluções que possam ser aplicadas.

\section{MÉ T O D O S}

Participaram da pesquisa 28 atletas competidores do sexo masculino, cujas características estão descritas na Tabela 1. A amostra foi selecionada de acordo com os seguintes critérios: (1) ter idade entre 16 e 29 anos; (2) estar participando, no momento da coleta, de torneios de nível regional ou mais importante; (3) ter graduação mínima de faixa-marrom; (4) praticar judô há, pelo menos, seis anos. Os atletas treinavam na cidade de São Paulo, local onde ocorreu a coleta de dados. O estudo foi conduzido entre agosto de 2002 e junho de 2004.

Após leitura e assinatura do termo de consentimento, os atletas foram pesados em uma balança digital (Kratos-CAS, modelo Línea) e medidos em estadiômetro de prancha. A composição corporal foi avaliada pelo método da pesagem hidrostática. Em seguida, eles responderam a um questionário de avaliação do nível competitivo e, então, foram entrevistados por nutricionista. Todo o procedimento foi aprovado pelo Comitê de Ética em Pesquisa com Seres Humanos do Instituto de Ciências Biomédicas da Universidade de São Paulo.

$\mathrm{Na}$ entrevista, os atletas responderam a um inquérito do tipo história alimentar ${ }^{12}$, no qual a nutricionista perguntou sobre o peso atual e sobre o histórico de peso (categoria em que competia, peso que costumava manter, peso máximo e mínimo atingido). Os atletas foram questionados também sobre quais métodos utilizaram para chegar ao peso mínimo, e em quais circunstâncias atingiram o peso máximo. Da mesma forma, eles relataram as estratégias que utilizavam para perder peso antes das competições.

Apenas 19 atletas compareceram à entrevista com a nutricionista. Dos outros 9 foram verificados apenas o nível competitivo, o peso corporal, e o peso da categoria.

Os dados foram analisados por meio de estatística descritiva, com auxílio do software SPSS 10.0, e estão apresentados em média (M) e desvio-padrão (DP).

\section{RESULTADOS}

A maioria dos sujeitos encontrava-se, no momento da entrevista, acima do peso máximo de sua respectiva categoria. Do total da amostra, 85,7\% (24 atletas) estava acima do peso, 10,7\% (3 atletas) estava abaixo do peso, e 3,6\% (1 atleta) estava, exatamente, no limite da categoria. Eles se encontravam, em média 4,2\% (2,9kg, DP=1,7kg) acima do peso da categoria. Os dados referentes ao percentual de indivíduos com excesso de peso, no momento da coleta, estão apresentados na Figura 1.

Tabela 1. Características gerais de judocas de elite $(n=28)$. São Paulo, 2004.

\begin{tabular}{|c|c|c|c|c|c|c|c|c|c|c|}
\hline \multicolumn{2}{|c|}{ Idade (anos) } & \multicolumn{2}{|c|}{ Peso $(k g)$} & \multicolumn{2}{|c|}{ Estatura $(\mathrm{cm})$} & \multicolumn{2}{|c|}{ Gordura corporal (\%) } & \multicolumn{2}{|c|}{ Tempo de judó (anos) } & \multirow{2}{*}{$\frac{\text { Nível competitivo (\% de amostra) }}{\text { Estadual Nacional Internacional }}$} \\
\hline$M$ & $\mathrm{DP}$ & $M$ & DP & $M$ & $\mathrm{DP}$ & $M$ & $\mathrm{DP}$ & $M$ & DP & \\
\hline 20 & 3 & 75,3 & 11,7 & 173 & 8 & 7,4 & 2,3 & 13 & 3 & 21 \\
\hline
\end{tabular}

M= média; DP: desvio-padrão. 


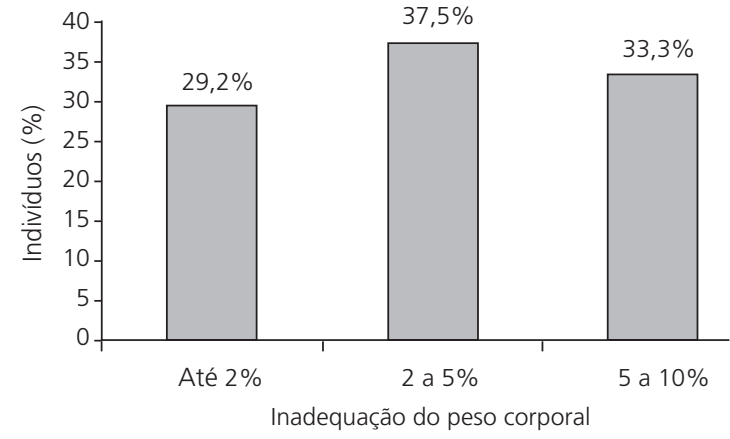

Figura 1. Judocas de elite com peso corporal acima do limite da categoria, de acordo com o índice de inadequação (n=24). São Paulo, 2004.
As estratégias mais relatadas para desidratar envolveram corridas ou treinos com agasalhos e sacos plásticos debaixo do kimono e restrição total ou parcial da ingestão de líquidos. Outros relatos bastante recorrentes foram: deixar de comer à noite, deixar de comer entre as refeições e jejuar antes da pesagem. É importante salientar que os atletas reduziam o peso em menos de sete dias, e que grande quantidade de peso era perdida nos três últimos dias antes das pesagens, as quais, geralmente, ocorrem no mesmo dia da competição, horas antes do início das lutas.

Tabela 2. Peso habitual e peso máximo de judocas de elite, relatados durante o inquérito alimentar (n=19). São Paulo, 2004.

\begin{tabular}{lccccc}
\hline \multirow{2}{*}{ Peso } & Acima do limite da categoria (\%) & \multicolumn{3}{c}{ Peso corporal acima do limite máximo da categoria } \\
\cline { 3 - 6 } & & $\mathrm{M}$ & $\mathrm{DP}$ & Mediana & Variação \\
\hline Habitual & 89,5 & 2,5 & $1,1 \mathrm{~kg}$ & $3 \mathrm{~kg}$ & 1 a $4 \mathrm{~kg}$ \\
& & 3,7 & $1,8 \%$ & $4,1 \%$ & 1,2 a $6,7 \%$ \\
Máximo & 100,0 & 5,5 & $2,6 \mathrm{~kg}$ & $5 \mathrm{~kg}$ & 2 a $12 \mathrm{~kg}$ \\
& & 7,8 & $3 \%$ & $8,3 \%$ & 2,7 a $12,3 \%$ \\
\hline
\end{tabular}

M= média; DP: desvio-padrão.

Dos 19 judocas que responderam ao inquérito alimentar, 17 relataram que costumavam manter-se acima do peso da categoria, e apenas 2 costumavam manter o seu peso no limite da mesma. Todos os entrevistados relataram que o menor peso atingido equivaleu ao peso máximo de suas categorias, ou seja, quando esses atletas estão em seu menor peso, eles estão no limite máximo da categoria. Vale dizer que o peso máximo dos mesmos era atingido quando ficavam por algum tempo sem treinar, seja em função de lesão ou de férias. Já o peso mínimo era alcançado somente antes das pesagens para as competições. A Tabela 2 apresenta os dados referentes ao comportamento de peso dos judocas entrevistados.

A Tabela 3 apresenta os dados relativos aos métodos de redução rápida de peso antes das competições. Os dados foram computados segundo a quantidade de atletas que utilizavam com freqüência determinado método. Como a maioria dos judocas costuma utilizar mais de intervenção, o número total de intervenções foi maior do que o de sujeitos entrevistados.
Tabela 3. Procedimentos adotados por judocas de elite para perda rápida de peso $(n=19)$. São Paulo, 2004.

\begin{tabular}{lcc}
\hline Método & Atletas (n) Amostra (\%) \\
\hline Desidratar & 13 & 68,4 \\
Diminuir a ingestão energética total & 12 & 63,1 \\
Cortar gorduras e "guloseimas" & 9 & 47,4 \\
Fazer mais exercícios & 5 & 26,3 \\
Cortar ou diminuir a ingestão no jantar & 5 & 26,3 \\
Aumentar a ingestão de salada & 5 & 26,3 \\
Diminuir a ingestão de carboidratos & 2 & 10,5 \\
\hline
\end{tabular}

D I S C U S S Ã O

Os resultados deste estudo indicam que judocas de elite têm dificuldades em se manter dentro dos limites de peso de suas respectivas categorias. Isso quer dizer que, momentos antes das competições, eles precisam reduzir o peso corporal, muitas vezes, em grandes quantidades, por meio de diversos métodos (Tabela 3), os quais são potencialmente danosos à saúde e ao desempenho ${ }^{1,3-8}$. 
O presente estudo constatou que a proporção da redução rápida de peso, a quantidade de peso a ser reduzida e as estratégias adotadas são semelhantes aos achados de Steen \& Brownell², Tipton \& Tcheng ${ }^{9}$ e Oppliger et al. ${ }^{10}$, os quais avaliaram atletas norte-americanos de luta olímpica. Da mesma maneira, os resultados obtidos sobre peso máximo atingido são bastante semelhantes aos valores de peso obtidos fora de temporada pelos referidos autores. Vale lembrar que os judocas deste estudo atingiram o peso máximo durante as férias ou durante um longo período sem treino.

Tipton \& Tcheng $^{9}$ observaram que o peso médio perdido entre o início da temporada e o período das competições foi de $3,1 \mathrm{~kg}(\mathrm{DP}=2,4)$ ou 4,9\% (DP=4). Os métodos mais utilizados para reduzir peso pelos atletas do referido estudo foram: diminuição do consumo de alimentos (16\%) e líquidos (14\%) e prática de exercícios em ambientes quentes $(23 \%)$ com roupas de plástico ou borracha (5\%). Steen \& Brownell ${ }^{2}$ verificaram que, entre competidores universitários, $41 \%$ variam seu peso de 5 a $9,1 \mathrm{~kg}$ por semana, enquanto $44 \%$ variam de 2,7 a 4,5kg por semana. Oitenta e nove por cento deles responderam que já tiveram de perder peso e que reduzem $4,4 \mathrm{~kg}(\mathrm{DP}=2,1)$ em 3 dias. Com relação à freqüência, 35\% responderam ter diminuído de 0,5 a $4,5 \mathrm{~kg}$ de peso mais de 100 vezes em sua vida, e $22 \%$ perderam entre 5 a $9,1 \mathrm{~kg}$ de 21 a 50 vezes durante a vida.

Embora os trabalhos citados anteriormente ${ }^{2,9}$ sejam relativamente antigos, os dados desta pesquisa demonstram que, a despeito das diferenças culturais entre Brasil e Estados Unidos, o comportamento atual com relação à perda de peso não apresenta diferenças importantes, principalmente se for considerado que tais estudos foram realizados há mais de 15 e 30 anos, respectivamente. A preocupação decorrente dessa constatação é que condutas realizadas há mais de 30 anos ainda são utilizadas por lutadores norte-americanos, embora em menor magnitu$\mathrm{de}^{13}$, mesmo com a implementação de programas educativos e as alterações nas regras ${ }^{10,13}$. Com relação aos atletas brasileiros, observa-se um padrão semelhante de comportamento, mas nenhum estudo foi ainda realizado e pouca importância tem sido dada ao assunto. A lógica competitiva impele os atletas a reduzirem o peso corporal por métodos perigosos e contraproducentes, como os observados neste trabalho.

Ainda em relação ao peso corporal dos judocas avaliados, apenas dois dos atletas haviam mudado para uma categoria mais pesada. Desses, um ainda estava 1,7\% acima do limite da nova categoria, enquanto o outro estava 1,4\% abaixo do peso máximo, embora tenha relatado manter-se constantemente 1,4\% acima desse peso. Isso indica que, apesar de não terem sido questionados a respeito disso, a freqüência com que os judocas entrevistados neste estudo reduzem peso é, provavelmente, elevada, já que eles competem sempre na mesma categoria, costumam pesar mais do que o limite da mesma, e participam de inúmeras competições por temporada.

Haja vista que tais práticas são absolutamente recorrentes, há um constante ciclo de redução e ganho rápido de peso (WC - weight cycling). Esse fato tem sido apontado como um agravante no quadro de inadequação do peso da categoria em relação ao seu peso corporal ${ }^{14}$, pois o rápido ganho de peso, após rápida redução, ocorre devido a adaptações fisiológicas pelas quais o corpo se torna mais eficiente na utilização e armazenamento de energia (aumento na eficiência alimentar). Aliada a esse aumento da eficiência alimentar, há uma diminuição da taxa metabólica basal, o que torna as próximas reduções cada vez mais difíceis ${ }^{14}$ e exige restrições energéticas cada vez maiores ${ }^{1}$.

Especula-se que a composição corporal possa modificar-se em decorrência da prática prolongada de WC. Ao retornar ao peso habitual, após o período de redução, é possível que haja aumento na quantidade de gordura corporal ${ }^{15} \mathrm{e}$ alterações na sua distribuição, em favor de maior acúmulo nas regiões centrais ${ }^{15,16}$. Especula-se, ainda, que a prática de WC possa aumentar as chances de problemas cardíacos, como hipertensão ${ }^{17}$. 
Conforme mencionado, apesar deste estudo não ter questionado diretamente os judocas a respeito da freqüência de redução de peso, pode-se inferir, pelo elevado número de competições durante uma temporada, e pelo fato de eles raramente "subirem" de categoria, que eles praticam WC. Esse fato é um indicativo da preocupante magnitude do problema de competir em categorias de peso que não condizem com a morfologia dos atletas.

Os principais efeitos adversos da perda rápida de peso, atingida por restrição alimentar e desidratação, são: (1) diminuição do volume plasmático e sangüíneo ${ }^{18 ;}$ (2) redução da função cardíaca, a qual está associada ao aumento da freqüência cardíaca, diminuição do volume de ejeção e do débito cardíaco ${ }^{19}$; (3) diminuição da concentração sérica de testosterona ${ }^{20 ;}$; 4 ) diminuição do fluxo sangüíneo renal e do volume de filtração glomerular ${ }^{21}$; (5) aumento da perda de eletrólitos ${ }^{22}$; (6) diminuição da atividade do sistema imunológico5,6; (7) piora do estado de humor ${ }^{4,23}$; (8) aumento do risco de transtornos alimentares ${ }^{24 ;}$; (9) e até mesmo interrupção temporária do crescimento ${ }^{25}$.

A rápida redução de peso pode prejudicar o rendimento competitivo, embora ainda permaneçam dúvidas a respeito disso. Há consenso no que se refere aos efeitos sobre o desempenho de endurance, o qual é diminuído pela rápida perda de peso ${ }^{26}$. No entanto, os efeitos da perda rápida de peso sobre o desempenho anaeróbio (o mais relacionado ao desempenho em lutas) são mais controversos. A despeito dessa contradição, é provável que as capacidades mais importantes para uma boa performance no judô sejam, de fato, prejudicadas 3,27,28.

Os principais mecanismos envolvidos com a redução do desempenho após uma rápida redução do peso corporal são: (1) diminuição dos estoques de glicogênio muscular causada pela restrição energética, combinada à prática de exercícios intensos ${ }^{27}$; (2) redução na taxa de utilização do glicogênio $7,18,23$; (3) perda aguda de massa muscular7,14,28; (4) desequilíbrio no sistema de tamponamento do bicarbonato e conseqüente aumento da acidose muscular, causados pela diminuição do conteúdo de glicogênio muscular ${ }^{23}$ e pela ceto-acidose metabólica ${ }^{27}$; (5) aumento da temperatura corporal, e maior dificuldade de termorregulação ${ }^{29}$.

Considerando os métodos utilizados pelos atletas deste estudo para redução do peso corporal, bem como a quantidade de peso reduzida, é possível que ocorra desidratação, perda de eletrólitos, depleção de glicogênio muscular e desequilíbrio na regulação ácido-base. Diante desse fato é provável que judocas brasileiros de elite compitam em condições metabólico-fisiológicas que tendem a diminuir a força e a potência muscular, assim como acelerar o processo de fadiga. Isso torna óbvia a relação negativa com a performance em lutas. Além disso, a constante utilização de tais métodos coloca em risco a saúde desses atletas.

Como recomendações práticas, para que se evite queda no desempenho, pode-se sugerir a adoção de dietas balanceadas para perda gradual de peso - ao invés de perda rápida - que não sejam acompanhadas por restrições hídricas e que contenham elevado teor de carboidra$\operatorname{tos}^{23,30,31}$. Pode-se recomendar, ainda, que o atleta consuma uma dieta composta basicamente por carboidratos nas horas compreendidas entre a pesagem e o início dos combates? ${ }^{7}$.

No entanto, mais do que, com essas recomendações, encorajá-los a continuar perdendo peso, é preciso modificar o sistema de classificação das categorias e de pesagem, de modo que não sejam mais necessárias, tampouco permitidas, grandes reduções no peso corporal.

Tcheng \& Tipton ${ }^{32}$ propuseram uma fórmula para predizer o peso mínimo no qual lutadores de luta olímpica poderiam competir, o que poderia ser também adaptado para campeonatos nacionais. Além disso, Tipton et al. ${ }^{33}$ afirmam que, ao aumentar o número de categorias de peso, diminui-se a prevalência da redução rápida de peso. É interessante notar que esses autores referiam-se a um sistema no qual havia 12 dife- 
rentes categorias de peso, e que nos campeonatos nacionais e internacionais de judô existem apenas 7 categorias de peso, incluindo a "peso-pesado". Para Tipton et al..$^{33} \mathrm{o}$ ideal seria que o estabelecimento das categorias de peso obedecessem ao seguinte critério: intervalos de 6 a 8 libras (aproximadamente, 2,7 a 3,6kg) até as categorias inferiores a 155 libras (aproximadamente, 70kg), e intervalos de 10 libras (aproximadamente, 4,5kg) até a categoria sem limite de peso.

Dados recentes de Davis et al..$^{13}$ mostraram que as medidas adotadas pela National Collegiate Athletic Association (NCAA) para reduzir a perda rápida de peso entre lutadores de luta olímpica foram, em certa medida, eficientes, e concluem que, talvez, a tradição de reduzir o peso esteja mudando. É necessário ressaltar, no entanto, que, ainda assim, a prevalência da perda rápida de peso é bastante alta, e que muitos atletas seguem utilizando métodos "extremos" para redução do peso.

Em relação à metodologia utilizada neste estudo, cabem aqui algumas considerações. Nos trabalhos que avaliaram a prevalência e a magnitude da perda rápida de peso citados nesta discussão, conduzidos em países desenvolvidos, geralmente, são utilizados entrevistas ou questionários padronizados. Tais questionários são desenvolvidos e validados em língua inglesa. Sabe-se, entretanto, que não é adequado utilizar esses instrumentos em outras línguas sem um processo minucioso de tradução e validação ${ }^{34}$. Assim, no momento deste estudo, não existia um questionário traduzido e validado para a língua portuguesa. Dessa forma, os autores optaram por obter os dados necessários a partir de entrevista, pois, caso alguma situação preocupante fosse constatada, ter-se-ia a fundamentação para a avaliação psicométrica de um questionário. Assim, em passos futuros, os autores pretendem trabalhar na validação de questionários sobre perda rápida de peso e o "comer transtornado" (disordered eating) em atletas.

A história alimentar, por sua vez, é o método mais antigo e testado de avaliação do consumo alimentar ${ }^{12}$. Uma de suas grandes vantagens é que ela não fornece apenas dados de consumo alimentar, mas também de comportamentos alimentares, que foram, justamente, os dados coletados neste estudo. Assim, é sabido que a história alimentar é o método de escolha, quando se pretende levantar informações mais profundas sobre alimentação ${ }^{35}$. Muitos autores consideram que a validade da história alimentar é alta, especialmente em relação aos outros métodos. Ela tem sido utilizada, inclusive, em populações clínicas que apresentam aspectos do "comer transtornado" semelhantes aos apresentados pela amostra deste estudo ${ }^{36,37}$.

A principal limitação deste estudo é o baixo número de sujeitos participantes, o que impede que os resultados obtidos sejam extrapolados para a população estudada. Entretanto, a amostra foi composta por judocas de elite, e a metodologia utilizada permite boa confiança e acurácia para o tipo dado coletado.

Algumas medidas preliminares podem diminuir a incidência de perda rápida de peso entre judocas, e poderiam ser estudadas pelas entidades que organizam torneios de judô. São elas: aumentar a quantidade de categorias de peso; realizar, aleatoriamente, exames (osmolaridade da urina ou bio-impedância, por exemplo) que verifiquem o estado de hidratação dos atletas no momento da pesagem, para eventuais sanções àqueles que estiverem acentuadamente desidratados; realizar as pesagens somente instantes antes das lutas, entre outras. Sem dúvida, há necessidade de estudos que verifiquem a eficiência dessas e de outras medidas, bem como de uma discussão profunda entre comunidade científica, atletas e entidades esportivas, antes que sejam adotadas em larga escala.

Estudos futuros deveriam envolver não apenas atletas de elite, mas de todos os níveis competitivos, sexo e idade, com um número amostral ampliado. Outros deveriam ser conduzidos, ainda, para que mudanças nas regras visando à diminuição da perda rápida de peso entre atletas possam ser, efetivamente, implementadas. 


\section{O N CLUS Ã O}

Pode-se concluir que os judocas de elite deste estudo costumam pesar mais do que o limite da categoria, tal qual relatado pela literatura com atletas norte-americanos de luta olímpica. Para adequar o peso, esses atletas utilizam métodos que podem, tanto piorar o desempenho em lutas, quanto ser prejudicial à saúde em vários aspectos. Como forma de minimizar o problema pode-se aumentar a quantidade de categorias de peso, verificar o nível de hidratação dos atletas no momento da pesagem e diminuir o lapso de tempo entre a pesagem e a luta. De qualquer forma, é necessário que se façam levantamentos mais abrangentes e complexos a respeito desse assunto, e que se estudem medidas que visem a diminuir a prevalência e a magnitude do problema.

\section{COLABORADORES}

G. ARTIOLI realizou a correção do projeto de pesquisa e de todo o desenho experimental, fez o contado com os atletas para participarem do estudo, analisou os dados e redigiu o texto; F. SCAGLIUSI participou na coleta de dados e na definição da metodologia, na discussão e redação do texto; $V$. POLACOW participou na coleta de dados e contribuiu na discussão dos dados e redação do texto; B. GUALANO auxiliou em todas as etapas do estudo desde a coleta de dados à análise e interpretação dos dados e na elaboração do texto. A.H. LANCHA JUNIOR participou em todas etapas de elaboração do projeto, definição do desenho experimental e dos métodos, análise e interpretação dos dados e redação do texto final.

\section{REFER Ê N CIAS}

1. Brownell KD, Steen SN, Wilmore JH. Weight regulation practices in athletes: analysis of metabolic and health effects. Med Sci Sports Exerc. 1987; 19(6):546-56.

2. Steen SN, Brownell KD. Patterns of weight loss and regain in wrestlers: has the tradition changed? Med Sci Sports Exerc. 1990; 22(6):762-8.

3. American College of Sports Medicine. Position Stand on weight loss in wrestlers. Med Sci Sports Exerc. 1996; 28(2):ix-xii.
4. Filaire E, Maso F, Degoutte F, Jouanel P, Lac G. Food restriction, performance, psychological state and lipid values in judo athletes. Int I Sports Med. 2001; 22(6):454-9.

5. Kowatari K, Umeda T, Shimoyama T, Nakaji S, Yousuke $Y$, Sugawara K. Exercise training and energy restriction decrease neutrophil phagocytic activity in judoists. Med Sci Sports Exerc. 2001; 33(04):519-24.

6. Ohta S, Nakaji S, Suzuki K, Totsuka M, Umeda T, Sugawara M. Depressed humoral immunity after weight reduction in competitive judoists. Luminescence. 2002; 17(3):150-7.

7. Rankin JW, Ocel JV, Craft LL. Effect of weight loss and refeeding diet composition on anaerobic performance in wrestlers. Med Sci Sports Exerc. 1996; 28(10):1292-9.

8. Zambraski EJ, Tipton CM, Tcheng $T$, Jordon HR, Vailas AC, Callahan AK. lowa wrestling study: chances in the urinary profiles of wrestlers prior to and after competition. Med Sci Sports Exerc. 1975; 7(3):217-20.

9. Tipton $C M$, Tcheng TK. lowa wrestling study: weight loss in high school students. JAMA. 1970; 214(7): 1269-74.

10. Oppliger RA, Steen SN, Scott JR. Weight loss practices of college wrestlers. Int I Sport Nutr Exerc Metabol. 2003; 13(1):29-46.

11. Little NG. Physical performance atributes of junior and senior women, juvenile, junior and senior men judokas. J Sports Med Phys Fitness. 1991; 31(4):510-20.

12. Thompson FE, Byers T. Dietary assessment resource manual. J Nutr. 1994; 124(11Suppl):2245S-317S.

13. Davis SE, Dwyer GB, Reed K, Bopp C, Stosic J, Shepanski M. Preliminary investigation: the impact of the NCAA Wrestling Weight Certification Program on weight cutting. J Strength Cond Res. 2002; 16(2):305-7.

14. McCargar LJ, Crawford SM. Metabolic and anthropometric changes with weight cycling in wrestlers. Med Sci Sports Exerc. 1992; 24(11): 1270-5.

15. Saris WHM. Physiological aspects of exercise in weight cycling. Am J Clin Nutr. 1989; 49(5Suppl): 1099-104.

16. Steen SN, Oppliger RA, Brownell KD. Metabolic effects of repeated weight loss and regain in adolescent wrestlers. JAMA. 1988; 260(1):47-50.

17. Lissner L, Odell PM, D'agostino RB, Stokes J, Kreger $\mathrm{BE}$, Belanger AJ, et al. Variability of body weight and health outcomes in the Framingham population. N Eng J Med. 1991; 324(26): 1839-44. 
18. Burge CM, Carey MF, Payne WA. Rowing performance, fluid balance, and metabolic function following dehydration and rehydration. Med Sci Sports Exerc. 1993; 25(12):1358-64.

19. Ribsil PM, Herbert WG. Effect of rapid weight reduction and subsequent rehydration upon the physiological working capacity of wrestlers. Res Quarterly. 1970; 41(4):536-41.

20. Strauss RH, Lanese RR, Malarkey WB. Weight loss in amateur wrestlers and its effects on serum testosterone levels. JAMA. 1985; 254(23): 3337-8.

21. Rowell LB. Human cardiovascular adjustments to exercise and thermal stress. Physiol Rev. 1974; 54(1):75-159.

22. Costill DL, Sparks KE. Rapid fluid replacement following thermal dehydration. J Appl Physiol. 1973; 34(3):299-303.

23. Horswill CA, Hickner RC, Scott JR, Costill DL, Gould D. Weight loss, dietary carbohydrate modifications, and high intensity, physical performance. Med Sci Sports Exerc. 1990; 22(4): 470-6.

24. Polivy J, Herman CP. Dieting and binging. A causal analysis. Am Psychol. 1985; 40(2):193-201.

25. Horswill CA, Park SH, Roemich JN. Changes in the protein nutritional status of adolescent wrestlers. Med Sci Sports Exerc. 1990; 22(5):599-604.

26. Fogelholm MG. Effects of bodyweight reduction on sports performance. Sports Med. 1994; 18(4): 249-67.

27. Hickner RC, Horswill JM, Welker J, Scott JN, Roemich JN, Costill DL. Test development for the study of physical performance in wrestlers following weight loss. Int J Sports Med. 1991; 12(6):557-62.

28. Klinzing JE, Karpowicz W. The effects of rapid weight loss and rehydration on a wrestling performance test. J Sports Med. 1986; 26(2): 149-56.
29. Bock WE, Fox EL, Bowers R. The effect of acute dehydration upon cardiorespiatory endurance. J Sports Med Phys Fitness. 1967; 7(2):62-72.

30. McMuray RG, Proctor CR, Wilson WL. Effect of caloric deficit and dietary manipulation on aerobic and anaerobic exercise. Int J Sports Med. 1991; 12(2):167-72.

31. Fogelholm MG, Koskinen R, Laskaso J, Rankinen T, Ruokonen I. Gradual and rapid weight loss: effects on nutrition and performance in male athletes. Med Sci Sports Exerc. 1993; 25(3):371-7.

32. Tcheng TK, Tipton CM. Iowa wrestling study: anthropometric measurements and the prediction of a "minimal" body weight for high school wrestlers. Med Sci Sports Exerc. 1973; 5(1):1-10.

33. Tipton CM, Tcheng T, Zambraski EJ. lowa wrestling study: weight classification systems. Med Sci Sports Exerc. 1976; 8(2):101-4.

34. Bowden A, Fox-Rushby JA. A systematic and critical review of the process of translation and adaptation of generic health-related quality of life measures in Africa, Asia, Eastern Europe, the Middle East, South America. Soc Sci Med. 2003; 57(7): 1289-306.

35. Kohlmeier L. Future of dietary exposure assessment. Am J Clin Nutr. 1995; 61(3suppl):702S-9S.

36. American Dietetic Association. Position of the American Dietetic Association: nutrition intervention in the treatment of anorexia nervosa, bulimia nervosa, and eating disorders not otherwise specified (EDNOS). J Am Diet Assoc. 2001; 101(7):810-19.

37. Van Binsbergen CJ, Hulshof KF, Wedel M, Odink J, Coelingh Bennink HJ. Food preferences and aversions and dietary pattern in anorexia nervosa patients. Eur J Clin Nutr. 1988; 42(8):671-8.

Recebido em: 10/8/2005

Versão final reapresentada em: 14/7/2006 Aprovado em: 20/12/2006 
